\title{
AN UNSHELLABLE TRIANGULATION OF A TETRAHEDRON
}

\author{
BY MARY ELLEN RUDIN
}

Communicated by R. H. Bing, February 14, 1958

A triangulation $K$ of a tetrahedron $T$ is shellable if the tetrahedra $K_{1}, \cdots, K_{n}$ of $K$ can be so ordered that $K_{i} \cup K_{i+1} \cup \cdots \cup K_{n}$ is homeomorphic to $T$ for $i=1, \cdots, n$. Sanderson [Proc. Amer. Math. Soc. vol. 8 (1957) p. 917] has shown that, if $K$ is a Euclidean triangulation of a tetrahedron then there is a subdivision $K^{\prime}$ of $K$ which is shellable; and he raises the question of the existence of a Euclidean triangulation of a tetrahedron which is unshellable. Such a triangulation will be described here.

Let $T$ be a tetrahedron each of whose edges has length 1 .

We will describe a nontrivial Euclidean triangulation $K$ of $T$ such that, if $R$ is any tetrahedron of $K$, then the closure of $(T-R)$ is not homeomorphic to $T$.

I. Construction of $K$ : Let $X_{1}, X_{2}, X_{3}$, and $X_{4}$ be the vertices of $T$.

The possible values for the letters $i$ and $j$ are 1, 2, 3, and 4 and addition involving $i$ or $j$ will be modulo 4 .

For each $i$, let $F_{i}$ denote the face of $T$ opposite $X_{i}$, and let $U_{i}$ be the midpoint of the interval $X_{i} X_{i+2}$. Observe that $U_{1}=U_{3}$ and $U_{2}=U_{4}$.

Let $\epsilon$ be the length of the shortest side of a triangle whose longest side is of length 1 and two of whose angles are $1^{\circ}$ and $60^{\circ}$.

For each $i$, let $Y_{i}$ denote the point of $F_{i+1}$ at a distance $\left(3^{1 / 2} / 2\right) \epsilon$ from $X_{i}$ such that the angle $Y_{i} X_{i} X_{i+2}$ is $1^{\circ}$.

For each $i$, let $Z_{i}$ denote the point of $F_{i+2}$ such that the angle $Z_{i} X_{i} X_{i+3}$ is $1^{\circ}$ and the angle $Z_{i} X_{i+1} X_{i}$ is $1^{\circ}$.

The fourteen vertices of our triangulation $K$ are the points $X_{i}, Y_{i}$, $Z_{i}$, and $U_{i}$. It can be shown that no triangulation which has less than 14 vertices has the desired property.

The tetrahedra of our triangulation $K$ are the tetrahedra of the forms:

(1) $X_{i} Z_{i} X_{i+1} Y_{i}$

(2) $X_{i} Z_{i+1} X_{i+1} Y_{i}$

(3) $Z_{i} Z_{i+1} X_{i+1} Y_{i}$

(4) $Z_{i} Z_{i+1} X_{i+1} Y_{i+1}$,

(5) $Z_{1} Z_{2} Z_{3} Z_{4}$,

(6) $Z_{i} Z_{i+1} Y_{i} Z_{i+2}$,

(7) $X_{i} Z_{i+1} Y_{i} Z_{i+2}$

(8) $X_{i} Z_{i+1} Y_{i+2} Z_{i+2}$

(9) $X_{i} U_{i} Y_{i+2} Z_{i+2}$ 
(10) $X_{i} U_{i} Y_{i} Z_{i+2}$

(11) $Z_{i} U_{i} Y_{i} Z_{i+2}$.

II. Checking the construction: The best method of doing this is to draw a big picture and label the vertices.

It is easy to check that for each tetrahedron $R$ of $K$, the closure of $(T-R)$ is not homeomorphic to $T$.

In order to check that $K$ is a triangulation, first observe that, for each $i$, the tetrahedra (1), (2), (3), and (4) fit together and form a thin rod having the triangles $X_{i} Y_{i} Z_{i}$ and $X_{i+1} Y_{i+1} Z_{i+1}$ for its ends; the union of these rods forms a torus running along the edges $X_{i} X_{i+1}$. When (5) is added to this torus the remainder of $T$ is divided into two congruent pieces each containing pieces of $T$ along the faces $F_{i}$ and $F_{i+2}$. After (6), (7), and (8) are added to the first five types there is only a small strip around $X_{i} X_{i+2}$ remaining of $T$; (9) and (10) complete the faces of $T$ and (11) fills in the final space.

To see that the tetrahedra all nest together properly in the order just described, the following facts will be useful. Fact $A$ is needed for the "rods." Fact B is needed for (3). Facts C and D are needed as assurance that none of the tetrahedra of types (5) through (11) intersect the interior of the torus. Fact $\mathrm{E}$ is needed to show that (7) does not intersect either (2) or (6). And facts F, G, and $H$ are needed to show that the tetrahedra of types (6) through (11) for $i=1$ do not intersect the tetrahedra of the same types for $i=3$. The facts can be easily proved using the definitions of $\epsilon, Y_{i}$, and $Z_{i}$.

(A) The plane $X_{i} Y_{i} Z_{i}$ separates $X_{i+1}, Y_{i+1}, Z_{i+1}$ from $X_{i-1}, Y_{i-1}$, and $Z_{i-1}$.

(B) The points $X_{i}$ and $Y_{i}$ are on the same side of the plane $X_{i+1} Z_{i} Z_{i+1}$.

(C) The plane $Y_{i} Z_{i} Z_{i+3}$ separates $X_{i}$ and $X_{i+3}$ from $U_{i}, X_{i+2}$, $Y_{i+2}, Z_{i+2}, X_{i+1}, Y_{i+1}$, and $Z_{i+1}$.

(D) The plane $Y_{i} Z_{i} Z_{i+1}$ separates $X_{i}$ and $X_{i+1}$ from $U_{i}, X_{i+2}$, $Y_{i+2}, Z_{i+2}, X_{i+3}, Y_{i+3}$, and $Z_{i+3}$.

(E) The plane $X_{i} Y_{i} Z_{i+1}$ separates $Z_{i}$ from $Z_{i+2}, X_{i+2}, Y_{i+2}$ and $U_{i}$.

(F) The plane $Z_{i} Z_{i+2} U_{i}$ separates $X_{i}, Y_{i}, Z_{i+1}, Y_{i+1}, X_{i+1}$ from $X_{i+2}, Y_{i+2}, Z_{i+3}, Y_{i+3}, X_{i+3}$.

(G) The plane $Y_{i+2} Z_{i+2} U_{i}$ separates $X_{i}$ and $Z_{i+1}$ from $X_{i+2}, X_{i+3}$, $Y_{i+3}$, and $Z_{i+3}$.

(H) The plane $X_{i} Z_{i+2} U_{i}$ separates $Y_{i+2}$ and $Z_{i+1}$ from $Y_{i}, Z_{i}$, and $Z_{\text {i+s. }}$

UNIVERSITY OF ROCHESTER 